\title{
Immanuel Wallerstein \\ Marx, der Marxismus-Leninismus und sozialistische Erfahrungen im modernen Weltsystem
}

Zusammenfassung: Im Gegensatz zum Marxismus hat die historische Rolle des Marxismus-Leninismus ausgespielt. Wallerstein betrachtet letzteren als vorübergehendes Phänomen in der historischen Entwicklung des modernen Weltsystems. Der Marxismus-Leninismus, der einer Ideologie nationaler Entwicklung stets näher stand als einer Ideologie sozialistischen Aufbaus, hat deshalb ausgedient, weil alle nationalen Entwicklungstheorien heute an ein Ende geraten sind.

Marx und seine Gedanken sind am Leben; sie stehen heute stärker da als die jedes anderen wissenschaftlichen Denkers des neunzehnten Jahrhunderts, und vermutlich werden sie auch für das gesellschaftliche Leben des Weltsystems im einundzwanzigsten Jahrhundert zentral bleiben. Der Marxismus-Leninismus als Strategie und Ideologie jedoch hat seine historische Rolle ausgespielt und ist für die politische Ökonomie des Weltsystems zu einer Randerscheinung geworden. Die sozialistische Praxis im modernen Weltsystem schließlich, wenn wir sie denn so bezeichnen können, befindet sich in großer Unordnung und wird vielleicht als »sozialistisch « erkennbar nicht überleben können.

Ich möchte hier den Marxismus-Leninismus als ein historisches Phänomen des modernen Weltsystems von seinen Ursprüngen bis heute analysieren, und zwar in Gestalt von Antworten auf sechs Fragen: 1. Warum Leninismus? 2. Warum zuerst in Rußland einen leninistische Revolution? 3. Warum Stalinismus? 4. Warum ein sowjetisches Imperium? 5. Warum Entstalinisierung? 6. Warum Perestroika und Glasnost?

\section{Antwort eins}

Als organisatorische Geburtsstunde des Marxismus-Leninismus oder Bolschewismus gilt gemeinhin das Jahr 1902, mit Lenins Gegenentwurf zu Plechanows Text für den zweiten Kongreß (den sogenannten Vereinheitlichungskongreß) der allrussischen sozialdemokratischen Partei im Sommer 1903. Wie wir wissen, führte der Kongreß zu einer Spaltung der Partei. Lenin erwies sich als hervorragender politischer Taktiker und ging aus dem Kongreß an der Spitze der Partei hervor, die sich (wenn auch einigermaßen umstritten) als die Mehrheit (Bolschewiki) bezeichnen konnte. Es ist heute zum Allgemeinplatz geworden, die Geschichte der sozialistischen Bewegungen in aller Welt als die Geschichte einer historischen Spaltung in zwei Tendenzen zu simplifizieren, die durch Eduard Bernstein und Lenin symbolisiert werden; organisatorisch wurde diese Spaltung nach 1921 durch die Existenz zweier Interna- 
tionalen, der Zweiten und Dritten, besiegelt. Eine andere Kurzformel für diese Entwicklung ist die Trennung von Reform und Revolution. Vereinfachungen sind immer angreifbar, aber für die erste Hälfte des zwanzigsten Jahrhunderts erscheint mir diese klassische Formel als im Wesen richtig.

Die Schlüsselargumente der »Revisionisten« leiteten sich von einem geradlinigen und ziemlich ökonomistischen Verständnis der Revolution des Weltsystems her. Sie sahen einen Prozeß unvermeidlichen technologischen Fortschritt, der eine immer größere Industriearbeiterklasse hervorbringen würde. Sie gingen dav on aus, dies werde politisch unvermeidlich zur Ausweitung politischer Rechte führen (insbesondere des Wahlrechts), vermutlich unter dem kombinierten Druck aus kapitalistischer Rationalität und Kämpfen der Arbeiterklasse. Sie argumentierten, im Laufe der Zeit werde die Klasse der Industriearbeiter zahlenmäßig die politische Arena dominieren, und so könnten sie sich recht einfach an die Macht wählen. Dann könnten sie dem Kapitalismus durch Gesetzgebung ein Ende bereiten und eine sozialistische Gesellschaft aufbauen. Aus dieser Argumentation leiteten sie die optimale politische Taktik ab, die darin bestand, einen möglichst großen Teil der Arbeiterklasse (sowie ihre Sympathisanten) politisch und gesellschaftlich in einer Massenpartei zu organisieren.

Die Argumentation war klar und überzeugte viele. Tatsächlich erwies sich dieses Drehbuch in der Realität als nur teilweise richtig. Es gab tatsächlich permanent technologischen Fortschritt, und der Umfang der Industriearbeiterklasse nahm zu. Das allgemeine Wahlrecht wurde zur Realität. Es stimmte jedoch nicht, daß die Industriearbeiterklasse eine große Mehrheit der Wahlbevölkerung bildete. Ebensowenig stimmten alle Arbeiter für die sozialistische Partei. Die Parteien der Zweiten Internationale kamen in einer ganzen Reihe von Ländern an die Macht. Sie konnten dem Kapitalismus jedoch nicht durch Gesetzgebung ein Ende bereiten. Sie schufen vielmehr den sogenannten Wohlfahrtsstaat.

Warum verschloß sich Lenin dieser Argumentation? Das lag daran, daß er andere Variablen in das Drehbuch einbrachte. Die erste und wichtigste Variable, auf der er bestand, war die Intensität, mit der die kapitalistischen Schichten sich ihrer Liquidierung widersetzen würden. Er ging davon aus, sie würden ihre bestehende Kontrolle der Staatsapparate dazu nutzen, mit allen Mitteln (fair oder unfair) für die Erhaltung ihrer Position zu kämpfen. Er hielt daher die Vorstellung, sie könnten aus der Macht gewählt werden, für eine völlige Schimäre. Deshalb bestand er darauf, der einzige Weg zur Macht der Arbeiterklasse liege in der Revolution, das heißt im Aufstand. Er erkannte diese Kämpfe als politisch-militärische und bestand einleuchtenderweise darauf, daß eine wesentliche Voraussetzung des Erfolgs eine hochdisziplinierte Organisation war. Ganz logisch folgten daraus seine Ansichten zur Parteistruktur. Da die Bourgeoisie alle Mittel einsetzen würde, um an der Macht zu bleiben, und da ein politisch-militärischer Kampf eine disziplinierte Organisation voraussetzte, sollte die Partei aus ergebenen und mehr oder weniger professionellen Parteiarbeitern bestehen, und zumindest teilweise sollte sie im Untergrund arbeiten. Auf diese Art, argumentierte er, konnte die Partei, wenn die Zeit reif war, die Macht ergreifen und eine sogenannte Diktatur des Proletariats errichten. Seine Vorstellung davon, was danach 
geschehen würde, unterschied sich jedoch nicht allzusehr von der der Revisionisten. Die neue Regierung würde dem Kapitalismus durch Gesetzgebung ein Ende machen und eine sozialistische Gesellschaft errichten.

Auch diese Argumentation war klar und überzeugte ebenfalls viele. Tatsächlich bewahrheitete sich aber auch dieses Drehbuch in der Realität nur teilweise. Aufstände waren in nur wenigen Ländern erfolgreich. In der Praxis erwies sich China als der Fall, der Lenins Vorstellungen am nächsten kam: Dort führte wirklich eine disziplinierte Kaderpartei einen langen politisch-militärischen Kampf, ergriff schließlich die Macht und errichtete eine Diktatur des Proletariats. Es ist richtig, daß die Parteien der Dritten Internationale, wo immer sie (mit welchen Mitteln auch immer) an die Macht kommen, per Gesetz den Kapitalismus beendeten (in dem engen Sinne der Abschaffung des Privateigentums an den meisten produktiven Unternehmen). Ob sie sozialistische Gesellschaften errichteten, ist seit 70 Jahren umstritten, ganz besonders in den letzten Jahren.

Lenin ging bei seiner Argumentation von einer nicht weiter ausgeführten Voraussetzung aus. Er kämpfte in Rußland und Rußland war nicht Deutschland, und schon gar nicht Großbritannien. Das soll heißen, daß Rußland ein Land war, in dem die Technologie, verglichen mit Westeuropa und Nordamerika, »rückständig « war. Darüber hinaus war Rußland ein Land, das nur für einen sehr kurzen Zeitraum überhaupt ein parlamentarisches System hatte, ganz zu schweigen von der Erwartung, es werde in absehbarer Zeit das allgemeine Wahlrecht einführen. In jedem Fall gab es nur sehr wenige Industriearbeiter, selbst wenn es das allgemeine Wahlrecht gegeben hätte. Die große Mehrheit der Bevölkerung bestand aus Landarbeitern und Bauern. Daher erschien das revisionistische Drehbuch als völlig irrelevant für die russische Situation und man muß zugeben, daß Lenin in seiner Einschätzung im Wesentlichen recht hatte. Als einzig gangbare Alternative erschien ihm daher sein eigenes Programm. Ob dies korrekt war oder nicht, ist im Rückblick schwer einzuschätzen, da sich die sozialistische Bewegung überall in die eine oder andere Richtung bewegte, entweder zum Revisionismus oder zum Leninismus (häufig, aber nicht immer, zum MarxismusLeninismus).

\section{Antwort zwei}

Es ist hinreichend bekannt, daß die russische Revolution von 1917 insoweit eine Überraschung bildete, als praktisch jedermann in der sozialistischen Weltbewegung den »ersten « Sozialistischen Staat in Deutschland erwartete. Diese Erwartung wurde auch von den Bolschewiki geteilt, selbst von Lenin. Warum niemand, sagen wir gegen 1900, daran glaubte, daß Großbritannien, Marx' eigener Kandidat, der erste sozialistische Staat sein würde, ist eine Frage, der nachzugehen sich lohnen würde, aber an dieser Stelle kann ich das nicht tun. Es zeigt jedoch, daß in der Argumentation sowohl der Revisionisten als auch der Leninisten andere Voraussetzungen versteckt waren. Wie wir wissen, fiel es Lenin im Oktober 1917 sehr schwer, seine Kollegen zu dem Versuch der Machtergreifung zu überreden. Und in jedem Falle schien das Gefühl 
verbreitet, die Abweichung einer der deutschen vorangehenden russischen Revolution werde alsbald korrigiert. In der Praxis argumentierten natürlich viele, der neue Sowjetstaat werde nicht überleben können, wenn nicht schnell etwas in Deutschland geschähe. Wir wissen, daß diese Erwartung niemals Realität wurde, und nach etwa fünf Jahren wurde sie schließlich aufgegeben.

Trotzdem: warum wurde diese Erwartung nicht erfüllt? E.H. Carr (1947, S. 105) beschreibt es bei seinem Versuch, die russische Revolution in eine historische Perspektive zu stellen, so:

»Die gleiche Ambivalenz, die die russische Geschichte des 19. Jahrhunderts durchzieht, beherrschte auch die bolschewistische Revolution. Einerseits war sie eine Kulmination des Verwestlichungsprozesses, andererseits eine Revolte gegen die europäische Durchdringung.«

1914 war Rußland ein europäisches Land, eine große Militärmacht und ein Land mit einem bedeutenden industriellen Sektor. Aber als Industrieland war es offensichtlich der schwächste der europäischen Staaten. 1914 war Rußland gleichzeitig ein nichteuropäisches (oder nicht westliches) Land, aber in erster Linie war es ein Agrarland. Als Agrarland wiederum war es offensichtlich der stärkste der nicht-westlichen Staaten. Daher war Rußland in unserer heutigen Sprache entweder die schwächste Macht im Kern oder stärkste der Peripherie. Natürlich war es beides - praktisch ein Beispiel für das, was wir heute ein semiperipheres Land nennen.

Ich möchte behaupten, daß eine leninistische Strategie nur in einem semiperipheren Land Erfolg haben konnte. Daher ist es in der Rückschau keineswegs überraschend, daß die erste sozialistische $\gg$ Revolution « in Rußland stattfand. Es war wahrscheinlich der einzige Ort, wo sie in jener Epoche möglich war. Dies war aus drei Hauptgründen offensichtlich. Der erste lautet, daß ein solcher Aufstand nicht in den Kernzonen der kapitalistischen Weltökonomie möglich war, weil die Arbeiterklasse zu viele Optionen hatte, die ihr kurzfristig attraktiver erschienen als die enormen Risiken eines Aufstands. Dies ist in den Jahren seit 1917 zweifellos klar geworden. Damals mag es nicht so offensichtlich erschienen sein.

Zweitens haben wir verstehen gelernt, daß die Mobilisierung der Massenunterstützung für eine Aufstandsbewegung ausschließlich auf der Grundlage der Beschwörung des Klassenbewußtseins nicht wahrscheinlich ist. Es muß dem Appell an das Klassenbewußtsein noch eine starke Dosis nationalistischer Gefühle hinzugefügt werden, die wir in der Folge als Anti-Imperialismus bezeichnet haben. Aber in der Kernzone hatten die meisten Länder das Bedürfnis nach nationalistischen Erfolgen hinter sich gelassen. Nur in Deutschland und Italien hätte dies ein größerer Faktor sein können, und natürlich waren dies in der Konsequenz die beiden Hauptorte faschistischer Bewegungen zwischen den Kriegen.

Drittens schließlich erfordert ein erfolgreicher Aufstand einen umfänglichen Sektor städtischer Infrastruktur, eine Arbeiterklasse und eine Intelligenzija von gewissem Umfang und Bewußtsein. Die meisten peripheren Zonen hatten dies 1917 noch nicht, aber Rußland schon. Daher erwies sich der Marxismus-Leninismus als die wirksame Ideologie für ein Vorgehen gegen das System in der Semipherie im Anfang des zwanzigsten Jahrhunderts, wie seine spätere Geschichte belegt hat. 


\section{Antwort drei}

Warum Stalinismus? Wiederum erscheint dies in der Rüickschau offensichtlich. Die Revolution war das Werk der Kaderpartei, definitionsgemäß einer kleinen Gruppe. Sie ging von der Perspektive aus, daß ihre Aufgabe politisch sehr schwierig sei, da sie die unnachgiebige Opposition der lokalen und der globalen Bourgeoisie zu überwinden hatte. Und tatsächlich bestätigte sich dies durch die Erfahrung der Sowjetunion, nicht nur in den ersten paar Jahren nach 1917, sondern auch in der Folge.

Zusätzlich zum Bürgerkrieg und zur ausländischen Intervention war Rußland ein vom Krieg verwüstetes Land, dessen ökonomische Stärke von vornherein nicht allzu groß gewesen war. Den Staat auch nur zusammenzuhalten, war eine monumentale Aufgabe, insbesondere da Rußland ein Imperium war und kein Nationalstaat. Der rapide Trend zum Einparteienstaat, zu einer merkantilistischen Politik des »Sozialismus in einem Land « und zur Umwandlung der Dritten Internationale zu einem weltweiten Unterstützungssystem für den belagerten »ersten sozialistischen Staat« sind sicherlich keine überraschenden Ergebnisse dieser Situation.

Das große Fragezeichen, wenn man sich die sowjetische Geschichte von heute aus vor Augen führt, steht hinter der Frage, ob den Bolschewiken nicht eine andere Haltung gegenüber der Bauernschaft mehr genützt hätte. Offensichtlich war die Zwangskollektivierung eine kritische Entscheidung und ein Wendepunkt, der eine Situation schuf, die sich noch heute auswirkt. Aber war sie unvermeidlich oder auch nur klug? Die marxistische Kultur hatte sicherlich die Sozialisten überall schlecht darauf vorbereitet, gegenüber der Bauernschaft eine politisch intelligente Haltung zu entwikkeln. Die Bauern galten, in den berühmten und berüchtigten Worten von Marx, als »ein Sack voll Kartoffeln«. Lenin, sollte man annehmen, hätte klüger sein können. Er hatte schließlich eine hervorragende Analyse der Entwicklung der agrarisch-ländlichen Gesellschaftsbeziehungen im späten zaristischen Rußland geschrieben. Er hatte zumindest, anders als Marx, die Bauernschaft studiert. Aber er war zu sehr in der manipulativen Politik eines Berufsrevolutionärs befangen, um seine einigermaßen akademischen Untersuchungen in Lektionen für politische Taktik zu übersetzen. Glaubte Lenin überhaupt an die NEP?

Tatsache ist, daß Rußland ein semiperipheres Land war; und seine Führung (und hier besteht eine Kontinuität von Witte bis Stalin) definierte die weltökonomische Priorität als die schnelle Industrialisierung des Landes. Lenins Slogan $\gg$ Kommunismus ist Sozialismus plus Elektrifizierung « hing noch bis vor wenigen Jahren auf großen Transparenten in Moskau. Die gesamte Führung glaubte an die »sozialistische Kapitalakkumulation « und rühmte sich bis in die achtziger Jahre ihrer großen Erfolge beim Anstreben dieses Ziels. Wenn Stahlwerke das ein und alles sozialistischer Planung sind, dann kann nicht allzuviel Sympathie existieren für die Leiden einer Bauernschaft, die enteignet und proletarisiert wird. Ein gelegentlicher taktischer Rückzug ja; eine grundlegende Berücksichtigung, niemals!

Heute ist es einfach, Bucharinist zu sein. Es ist weniger klar, ob Bucharins moderate Politik politisch durchführbar war. In jedem Fall setzte sie sich nicht durch. Ich glau- 
be, der entscheidende Grund dafür liegt darin, daß die Mehrheit der politisch aktiven Elemente in den zwanziger Jahren nicht iiberzeugt war, daß das Überleben des Sowjetstaates mit seiner Politik möglich war. Aber natürlich schuf die Zwangskollektivierung Bedingungen, die direkt (wenn auch vielleicht nicht unvermeidlich) zum Terror und den Säuberungen führten.

Der Stalinismus wurde darüber hinaus durch die Weltpolitik gestärkt. Im Zeitraum von 1933 bis 1941 war offensichtlich, daß sowohl Deutschland wie auch das westliche Trio (die Vereinigten Staaten, Großbritannien und Frankreich) nach einem neuen Weg suchten, wie der Sowjetstaat zerstört werden konnte. Stalins Argument, seine Politik sei defensiv gegenüber einem äußeren und mächtigen Feind, sie sei die einzige Möglichkeit, den Sowjetstaat zu verteidigen, mag nicht richtig gewesen sein, aber es überzeugte viele und gab dem Stalinismus in einem wichtigen Sinne einige Legitimität in der Öffentlichkeit. Der Zeitraum des Großen Vaterländischen Krieges verstärkte diese Legitimation natürlich enorm.

Darüber hinaus gab es eine weitere externe Unterstützung, die wir nicht vergessen sollten. Wir nehmen alle zu selbstverständlich an, es habe in der US-Politik eine Wende gegeben, von der Rooseveltschen Anpassungspolitik zu der Feindschaft Trumans und seiner Nachfolger im Kalten Krieg. Ich stimme dem nicht zu. Mir scheint, die USPolitik zeigte hinter allen rhetorischen Veränderungen eine Kontinuität. Die USA wollten eine stalinistische Sowjetunion mit einem Klein-Imperium, vorausgesetzt sie hielt sich im wesentlichen innerhalb der Grenzen von 1945-48. Der Stalinismus diente den USA als ideologische Rechtfertigung und Zement für ihre Hegemonie im Weltsystem. Stalinismus war ein schwächender, kein verstärkender Faktor für die weltweiten antisemitischen Kräfte. Stalinismus garantierte die Ordnung in einem Drittel der Welt, und die Sowjetunion konnte in diesem Sinne als eine »subimperialistische« Macht für die USA betrachtet werden. Vom Standpunkt der USA ist in der Sowjetunion seit dem Tode Stalins alles schlechter geworden. Man bedenke die gegenwärtige Unruhe, die das Phänomen Gorbatschow in den USA hervorruft.

\section{Antwort vier}

Warum schuf die Sowjetunion nach 1945 ein »Imperium«? Untersuchen wir genau, was sie wirklich tat. Es steht außer Frage, daß die Sowjetunion und die Rote Armee kommunistische Parteien in sechs Staaten an die Macht brachten, wo sie es auf andere Weise nicht geschafft hätten, weder durch Wahlen, noch durch Aufstand. Bei den sechs Ländern handelt es sich bekanntermaßen um Polen, Bulgarien, Rumänien, Ungarn, die Tschechoslowakei und die DDR.

Ich denke, die Erklärungen für dieses Vorgehen sind simpel, sogar banal. Zunächst fürchtete die Sowjetunion ein mögliches militärisches Vorgehen der USA und ein Wiederauferstehen Deutschlands, und sie wollte ihre militärische Position verstärken. Tatsächlich war dies zwar eine Fehlinterpretation der US-Strategie, aber sie wurde dennoch geglaubt. Zweitens wollte (und brauchte) die Sowjetunion wirtschaftliche 
Reparationsleistungen und hatte das Gefühl, der einzige Weg, sie sich zu verschaffen hieße, sie sich zu nehmen. Und drittens fürchtete die Sowjetunion tatsächlich die potentielle Stärke (und daher Unabhängigkeit) einheimischer kommunistischer Bewegungen und wollte sicherstellen, daß die osteuropäischen Parteien Satellitenparteien sein würden.

Natürlich mußte diese Art, kommunistische Parteien an die Macht zu bringen, jede Legitimation zerstören, die sie 1945 gehabt haben mochten. Die einzige mögliche (und zeitweilige) Ausnahme war die Tschechoslowakei, wo die Kommunistische Partei über eine echte lokale Stärke verfügte. Die Säuberungen von 1948-49 waren tatsächlich antinationalistisch, nicht anti-bourgeois-nationalistisch, sondern anti-kommunistisch-nationalistisch. Mit den Säuberungen war das Feigenblatt gefallen, und es war nur noch eine Frage der Zeit, daß nationalistische (und daher antisowjetische) Gefühle in politisch wirksamer Form wiederaufleben würden.

Im Gegensatz dazu hatten die Sowjetunion und die Rote Armee nichts zu tun mit der kommunistischen Machtübernahme der kommunistischen Guerilla-Bewegungen in Jugoslawien, Albanien und China. Und es ist daher kein Zufall, daß alle drei kommunistischen Regierungen in der Nachkriegszeit ziemlich spektakulär und offen mit der Sowjetunion brachen. Keine der drei war jemals ein Satellit oder Teil des sowjetischen »Imperiums«. Für kurze Zeit waren sie Verbündete, aber nicht mehr. Stalin verstand das von Anfang an. Deshalb riet er der chinesischen KP, sich mit der Kuomintang zu einigen, ein Ratschlag der ignoriert wurde. Deshalb verhinderte er die Anfänge einer jugoslawisch-bulgarischen Föderation, auf die Dimitroff hinarbeitete. Und deshalb natürlich zogen sich sowjetische Truppen 1946 aus dem nördlichen Iran zurïck, und deshalb ließ die Sowjetunion 1947 den kommunistischen Aufstand in Griechenland fallen. Stalin war nicht nur kein Freund, sondern ein offener Gegner einheimischer, national legitimierter kommunistischer Machtïbernahmen.

Sicherlich hat sich das »Imperium « ebenso als Bürde wie als Segen erwiesen, aber es ist keine Last, derer sich die Sowjetunion so einfach entledigen könnte. Nach 1968 und der Invasion in der Tschechoslowakei haben wir uns angewöhnt, von einer Breschnew-Doktrin zu sprechen, was die Unveränderlichkeit des Satelliten-Status bezeichnet. Sollten wir nicht besser von der Breschnew-Johnson-Doktrin sprechen? Gab nicht Johnson Breschnew die erforderlichen Absicherungen? Und wenn ja, warum? Die Antwort erscheint mir klar. Die USA wollten, daß die Sowjetunion auch weiterhin die Bürde des Imperiums trug (mit allen Lasten und Vorteilen), und sie wollten sicherlich weder das Risiko der Unordnung eingehen noch die ökonomischen Kosten bei einer erfolgreichen Emanzipation der Satelliten tragen. Johnson zögerte das Unvermeidliche um zwanzig Jahre hinaus. George Bush windet sich heute unter den Schwierigkeiten, die ihm die endlich in Gang gekommene Entwicklung bereitet.

\section{Antwort fünf}

Warum Entstalinisierung? Heute erscheint das als eine seltsame Frage. Wer mag schon Stalinismus? Offensichtlich würde ihn doch jeder abschaffen wollen. Wir müs- 
sen uns aber daran erinnern, daß es noch Mitte der achtziger Jahre viele Analytiker innerhalb und außerhalb der sozialistischen Länder gab, die dies für einen unmöglichen Gedanken hielten. Manche Leute trugen sogar vor, Chruschtschows Rede und der Bruch zwischen der Sowjetunion und China seien bloße Tricks oder Mllusion. 1953, in den Wochen nach Stalins Tod, begann Isaac Deutscher (1953) ein Buch zu schreiben, das »einen Bruch mit der Stalin-Ära« voraussagte. Wie er im Vorwort schreibt: »Meine Freunde, unter ihnen hervorragende Fachleute für sowjetische Angelegenheiten, wiegten skeptisch die Köpfe.«Der Kern von Deutschers Prognose lag in einem Satz: $\gg$ Der ökonomische Fortschritt, der während der Stalin-Ära erreicht wurde, hat endlich ein Maß an Wohlstand in die Reichweite des Volkes erbracht, der eine ordnungsgemäße Überwindung des Stalinismus und eine schrittweise demokratische Entwicklung möglich machen sollte« (1953, S. 221).

Deutscher gebührt heute viel Anerkennung für die grundlegende Vision, aber er hatte dann doch nur teilweise recht. Was Deutscher vorausgesagt hatte, wurde als Chruschtschowismus Praxis, und der Chruschtschowismus war ein Mißerfolg. Chruschtschow scheiterte nicht an seinem aufbrausenden Temperament, sondern weil er noch immer dem Entwicklungsdenken verhaftet war, das der sowjetischen Wirtschaftspolitik immer zugrunde gelegen hatte. Im Jahr 2000 werden wir euch beerdigen, sagte er den Amerikanern. Heute erscheint das wie eine Farce. Damals war es das jedoch nicht, und wir sollten analysieren, warum. Die Bemerkung erfolgte während des Zeitraums unglaublicher Expansion der kapitalistischen Weltwirtschaft, etwa von 1945 bis 1967. Damals »entwickelten « sich alle, aber einigen gelang es besser als anderen. Die Wachstumsraten in den Comecon-Ländern waren bemerkenswert hoch, und hochgerechnet hätte die Sowjetunion die USA wenn nicht im Jahre 2000 , dann doch ein oder zwei Jahrzehnte später »einholen« können.

Diese Wachstumsraten waren darüber hinaus nicht nur hoch im Vergleich zu den industriellen Kernländern, sondern sahen ganz besonders gut aus, wenn sie mit Ländern der Dritten Welt verglichen werden. Natürlich hatte auch Japan bemerkenswerte Wachstumsraten, aber in den fünfziger Jahren nahm das niemand zur Kenntnis. Das sowjetische Entwicklungsdenken war daher nicht nur eine Quelle des Stolzes für die kommunistischen Parteien an der Macht, sondern auch ein Leitstern für die nationalen Befreiungsbewegungen in der Dritten Welt. In den fünfziger Jahren war der Glaube an die Sowjetunion als ein Modell ökonomischer Entwicklung zwar nicht allgemein, aber doch weit verbreitet. Und die Erklärung für diesen Erfolg - insbesondere, aber nicht ausschließlich in der Dritten Welt - lag in der Leistungsfähigkeit des Leninismus. Ich sage eher Leninismus als Marxismus-Leninismus, weil viele Bewegungen in der Dritten Welt es teilweise aus Nützlichkeitsgründen, teilweise aus kulturellem Widerstand vorzogen, den Leninismus (insbesondere die Parteistrukturen und die staatliche Planung) zu übernehmen, ohne den Marxismus (insbesondere den Begriff eines internen Klassenkampfes und den Eurozentrismus) mit zu importieren.

Chruschtschow war kein Neuerer im Hinblick auf sein Entwicklungskonzept, tatsächlich setzte er das Erbe Stalins und Lenins fort. Seine Neuerung lag darin. daß er die Interessen der Kader des Sowjetsystems zu vertreten suchte, die zweierlei wollten: 
eine Garantie gegen den Terror und mehr Konsum. Seine Naivität lag letztlich in seiner Überzeugung, er könne den Prozeß der Liberalisierung ohne eine Reform der grundlegenden politischen Strukturen unter Kontrolle halten. Chruschtschowismus stand für eine grundlegende Unterschätzung der soziologischen Transformation der Sowjetunion, ebenso wie ein falsches Verständnis für das Funktionieren des modernen Weltsystems. Chruschtschowismus verkörperte auf gewisse Weise den Glauben an die sowjetische Rhetorik, eine Sünde, deren sich Stalin niemals schuldig gemacht hatte.

Die höheren Kader, die das wollten, was Chruschtschow zu bieten hatte, waren entsetzt, sobald sie erkannten, daß sie möglicherweise einen Geist aus der Flasche ließen. Breschnewismus war der Versuch, den Geist zurückzustopfen. Wie wir wissen, war der Versuch vergeblich. Die beiden Elemente, die Chruschtschow nicht berïcksichtigte, waren das Ausmaß der Verstädterung und der Veränderung der Arbeitskräfte in der Sowjetunion, sowie die zyklischen Rhythmen der kapitalistischen Weltwirtschaft. Als er in der Sowjetunion (und damit auch in den Comecon-Ländern) die Möglichkeit einer gewissen politischen Liberalisierung und eines gewissen Konsums eröffnete, unterschätzte Chruschtschow in verheerendem Maße die Nachfrage. Es ist seit langem eine Banalität der soziologischen Analyse, daß es für einen Staat einfacher ist, völlig repressiv zu sein, als einen kleinen, aber nicht ausreichenden Spielraum für politischen und kulturellen Pluralismus zu bieten. Ein kleiner Spielraum facht den Appetit an, ohne ihn zu befriedigen, und ermutigt dazu, mehr zu fordern. Wieviel Spielraum gebraucht wird, bevor wieder politische Ruhe eintritt, ist schwer einzuschätzen, aber augenscheinlich bot Chruschtschow zu wenig. Die Breschnew-Lösung war offensichtlich ein Schritt in die entgegengesetzte Richtung, ohne jedoch einen direkt lebensbedrohenden Terror einzuführen, der die höheren Kader wieder in Angst versetzt hätte. Eine solche Gegenpolitik kann eine Weile Erfolg haben und hatte unter Breschnew auch Erfolg, sowohl in der Sowjetunion wie in Osteuropa.

Aber Chruschtschow beging einen noch größeren Fehler. Er erkannte nicht, wie die kapitalistische Weltwirtschaft wirklich funktioniert. Die eindrucksvollen Wachstumsraten gründeten sich in erster Linie auf eine nicht dauerhaft leistungsfähige Grundlage extensiven Wachstums mit hoher Arbeitsintensität. Für eine Weile, und solange die Weltwirtschaft expandierte, konnte dies zu einem Wachstum des Bruttosozialprodukts führen, selbst des Bruttosozialprodukts pro Kopf. Aber die Ineffizienz der Arbeitsprozesse brachte es mit sich, daß die Zuwachsraten einen Gipfel erreichten, und daß hinter diesem Punkt der Zuwachs im Lebensstandard immer hinter dem Zuwachs in den industriellen Kernländern im gleichen Zeitraum hinterherhinkte, wenn auch nicht hinter dem in den meisten peripheren Zonen. Früher oder später konnten die sozialistischen Wirtschaften nicht mehr den Erwartungen auf Besserung bei einer immer größeren Schicht von Menschen genügen, die ausreichend gut informiert waren, um die Diskrepanzen zu erkennen. Natürlich war dies in den leistungsstärkeren Wirtschaften wie der DDR und der Tschechoslowakei nicht so ernst, aber selbst hier war es nur eine Frage der Zeit, bis der Überschuß die politisch-reale Nachfrage nicht mehr befriedigen konnte. 
Als der Abwärtsschwung in der Weltwirtschaft eintrat, unterschieden sich die Wirtschaftsprozesse in den sozialistischen Ländern nicht so sehr von denen der Dritten Welt. In den siebziger Jahren zogen einige Länder aus dem Ölpreis Nutzen, und die Sowjetunion gehörte dazu. Westliche Finanzinstitutionen drückten all diesen Ländern Kredite auf, um die effektive Weltnachfrage aufrechtzuerhalten, und nicht wenige sozialistische Länder wurden zu den größten Pro-Kopf-Schuldnem, um in den achtziger Jahren unter einer radikalen Unfähigkeit im Schuldendienst zu leiden, ganz zu schweigen von einer Rückzahlung (oder wenn sie zurückzahlten, so taten sie dies unter den unglaublichen sozialen und menschlichen Kosten wie sie etwa Rumänien sich aufgeladen hat). Und sozialistische Länder fanden sich nicht weniger als Drittwelt-Länder in großen Schwierigkeiten, ihre Produkte auf dem Weltmarkt zu verkaufen, im gegenwärtigen Jargon: »konkurrenzfähig« zu sein. Daher litten die sozialistischen Länder nicht weniger als die Länder der Dritten Welt unter Inflationsdruck und einem sinkenden Lebensstandard. Wie die meisten der Drittwelt-Länder begannen die sozialistischen Länder, Zuflucht in einer Liberalisierung ihrer Märkte zu suchen (oder wurden gedrängt, dort Zuflucht zu suchen). Und wie den meisten DrittweltLändern hat die zunehmende Öffnung der Märkte auch den sozialistischen Ländern bestenfalls eine leichte Linderung ihrer ökonomischen Schwierigkeiten verschafft.

\section{Antwort sechs}

Wir können Perestroika und Glasnost als konjunkturelle Reaktionen auf ein allgemeines Dilemma betrachten, und tatsächlich habe ich es soeben auf diese Weise beschrieben. Aber sie sind mehr als dies. In der Verkleidung einer Rückkehr zum Leninismus sind sie ein Versuch der Eliten, sich im Gefolge des weltweiten Zusammenbruchs des (Marxismus-)Leninismus als einer Ideologie und Strategie neu zu gruppieren. In diesem Prozeß beginnt sich die Sowjetunion endlich zu dekolonisieren (nicht nur innerhalb des sozialistischen Lager, sondern auch in ihren eigenen Grenzen).

Jeder spricht von Gorbatschows Schwierigkeiten: dem Dilemma einer Perestroika, die noch nicht funktioniert; dem Dilemma einer Glasnost, die noch nicht weit genug gegangen ist, um die meisten zu befriedigen, aber weit genug, um beträchtliche innere Probleme auszulösen; und schließlich dem Dilemma einer Dekolonialisierung, die à la de Gaulle oktroyiert wird, ohne das günstige ökonomische Weltklima, aus dem de Gaulle Nutzen zog. All dies ist wahr, aber in meiner Sicht von sekundärer Bedeutung. Gorbatschows hauptsächliches Dilemma liegt darin, daß er über keine alternative Ideologie und Strategie verfügt, um den ausgedienten Marxismus-Leninismus zu ersetzen. Er ist sicherlich ein hervorragender Taktiker. Er liquidiert den kalten Krieg tatsächlich ganz allein, und tut damit mehr, um der Entwicklung der Welt und der Sowjetunion eine positive Richtung zu garantieren, als jeder andere Führer der Gegenwart. Aber was ist letzten Endes mit dem sozialistischen Projekt passiert? Ich selbst glaube, daß wir die sozialistischen Erfahrungen unter der Herrschaft des Marxismus-Leninismus neu einschätzen müssen, sie in erster Linie als ein historisch 
verständliches, aber vorübergehendes Phänomen in der historischen Entwicklung des modernen Weltsystems begreifen müssen. Nicht daß sie gescheitert wären. Der Begriff $\gg$ Scheitern « geht davon aus, es habe plausible historische Alternativen gegeben. Ich glaube, es gab keine solchen plausiblen Alternativen zur Sozialdemokratie, die sich in der westlichen Welt entwickelte, zum Marxismus-Leninismus, der sich zumindest in der Sowjetunion und China festsetzte, und zu den nationalen Befreiungsbewegungen, die in der Dritten Welt an die Macht kamen. Dieser ganze Prozeß gilt für etwa ein Jahrhundert: von den siebziger Jahren des 19. Jahrhunderts, als diese Bewegungen real geboren wurden, bis 1968, das ich als den symbolischen Wendepunkt in der Geschichte dieser Bewegungen begreife (vgl. Wallerstein 1989).

Die drei Arten von Bewegungen repräsentierten tatsächlich lediglich drei Varianten einer einzigen Strategie: die Erringung der Macht im Staate durch eine Partei, die von sich behauptete, den öffentlichen Willen des Volkes zu verkörpern, und die die Staatsmacht einsetzte, um das Land zu »entwickeln«. Diese Strategie hat sich als unbrauchbar erwiesen, aber 1870 war dies noch nicht zu erkennen, noch nicht einmal 1945. Den Bewegungen sollte nicht zum Vorwurf gemacht werden, daß sie Produkte ihrer Zeiten waren. Aber heute leben wir in einem anderen Klima. René Dumont hat 1983 gesagt: Fini les lendemains qui chantent. Ich selbst glaube jedoch nicht, daß der Utopismus an seinem Ende angelangt ist. Ganz im Gegenteil. Vielleicht können wir erst heute utopische Utopien erfinden. ${ }^{1}$

Der Aufbau des Sozialismus in dieser Welt, wenn er denn stattfinden sollte, steht immer noch vor uns - als Option, aber kaum als Gewißheit. Die sogenannten real existierenden sozialistischen Erfahrungen können uns viel durch ihr negatives Beispiel lehren, und ein bißchen durch ihr positives Beispiel. Es ist gut sich zu erinnern, daß letzten Endes der Marxismus-Leninismus in der Realität eher als Ideologie nationaler Entwicklung denn als Ideologie des sozialistischen Aufbaus funktionierte. Nationale Entwicklung ist allerdings ihrem Wesen nach ein illusorisches Konzept innerhalb des Rahmens einer kapitalistischen Welt-Ökonomie ${ }^{2}$. Sie wird von den meisten Ländern niemals erreicht werden, nicht einmal teilweise. Der Marxismus-Leninismus hat als Ideologie heute ausgedient, weil alle Entwicklungsideologien ausgedient haben. Der Marxismus jedoch begann nicht als eine Ideologie nationaler Entwicklung und er ist nicht dazu verurteilt, nur in dieser begrenzten Art verstanden zu werden. Man kann Marx auch auf andere Art lesen. Und in den nächsten Jahrzehnten kann es, ja wird es wahrscheinlich noch viel Theorie und Praxis geben, die uns erlauben, zu einem neuen ideologischen Konsens zu gelangen, zu einer neuen wissenschaftlichen Epistemologie, einer neuen Historiographie, die Marx' fundamentale Einsichten und Werte beinhalten und auf marxistische Weise über sie hinausgehen zu einer neuen Aufhebung, die den Aufbau einer demokratischeren, egalitären Welt erlauben könnte. 


\section{Anmerkungen}

1 Ich habe schon früher das Verhältnis zwischen Marxismen und Utopien diskutiert. In diesem Artikel schrieb ich: Utopien sind immer ideologisch. Hier hatten Engels (und Marx) recht, voraus gesetzt, man erinnert sich, daß sie darin Unrecht hatten, daß sie implizit an die Utopie glaubten, es könne jemals ein Ende der Geschichte geben, an eine Welt, in der es Ideologien nicht mehr geben wird. Wenn wir einen Fortschritt erzielen sollen, dann, so scheint mir, miissen wir nicht nur den Widerspruch als Schlüssel zur Erklärung sozialer Wirklichkeit akzeptieren, sondern auch seine fortdauernde Unausweichlichkeit, eine Annahme, die orthodoxen Marxisten fremd ist. Der Widerspruch ist die Bedingung menschlichen Lebens. Unser Utopia muß nicht in der Ausschaltung aller Widersprüche gesucht werden, sondern in der Beseitigung der vulgären, unnötigen Konsequenzen materieller Ungleichheit. Dies letztere erscheint mir intrinsisch ein durchaus erreichbares Ziel. (1986, S. 1307)

2 Ich erörtere dies mehr im Detail in: Wallerstein (1988)

\section{Literatur}

Carr, E:H: (1947): The Soviet Impact on the Western World, New York

Deutscher, I. (1953): Russia - What next?, New York

Wallerstein, I. (1986): Marxism as Utopias: Evolving Ideologies, in: American Journal of Sociology, XCI, 6 May, 1295-1308

Wallerstein, I. (1988): Development: Lodestar or Illusion?, in: Economic and Political Weekly, XXIII, 39, Sept. 24, $2017-2023$

Wallerstein, I. (1989): 1968, Revolution in the World-System: Theses and Queries, in: Theory and Society, XVIII, $431-449$ 